\title{
Average-ILC-Based Consensus Tracking of Multiagent Systems over Wireless Networks in Presence of Channel Noise and Data Dropout
}

\author{
Chenlong Li, Yong Fang (D), and Zhichao Sheng \\ Shanghai Institute for Advanced Communication and Data Science, \\ Key laboratory of Specialty Fiber Optics and Optical Access Networks, \\ Joint International Research Laboratory of Specialty Fiber Optics and Advanced Communication, Shanghai University, \\ Shanghai 200444, China \\ Correspondence should be addressed to Yong Fang; yfang@staff.shu.edu.cn
}

Received 15 May 2020; Accepted 18 May 2021; Published 7 June 2021

Academic Editor: Elisa Francomano

Copyright (C) 2021 Chenlong Li et al. This is an open access article distributed under the Creative Commons Attribution License, which permits unrestricted use, distribution, and reproduction in any medium, provided the original work is properly cited.

\begin{abstract}
In a multiagent system (MAS), communication signals are affected by harsh wireless networks when they are transmitted from an agent to its neighboring agents, leading to the inconsistency of the MAS. In this paper, an average-iterative learning control (average-ILC) method is studied to address the consensus problem of MAS over wireless networks in the presence of channel noise and data dropout. The combined effects of channel noise and data dropout on iterative learning controllers are carefully analyzed. Based on graph theory and mathematical expectation, the corresponding average-iterative learning scheme is proposed. Especially, a sufficient condition is derived for the average-iterative learning scheme. Rigorous theoretical analysis demonstrates that the convergence of the covariance matrix of tracking error can be guaranteed with the help of an average-iterative learning scheme. Finally, simulation results are given to show the effectiveness of the proposed method.
\end{abstract}

\section{Introduction}

The consensus problem has become one of the most popular research studies in collaborative control of MAS [1-4]. Its purpose is to make each agent of MAS cooperate with others to achieve the desired trajectory and then complete a certain task. Meanwhile, ILC is an effective technology for dynamic systems that operate repetitively because it realizes the control tasks of dynamic systems with high uncertainty in a limited time interval. Thereby, ILC has attracted widespread attention in many areas, such as robotics [5], vehicles [6], industrial automation [7], and batch processes [8]. For these applications of ILC, it can be seen that ILC has a good performance in collaborative tasks. Thus, it is a splendid alternative to apply ILC algorithms in MAS.

The applications of ILC in multiagent frameworks have been developed for many years. ILC was firstly introduced into MAS by Ahn and Chen in [9], and the control input sequence of MAS was iteratively generated to realize formation control. Motivated by it, distributed ILC was proposed for multiagent tracking. In References [10, 11], the authors proposed distributed-ILC methods, which used the relation between local error and global error to solve the tracking problem of continuous-time systems and discrete-time systems, respectively. After that, Meng and Jia [12] extended distributed ILC for MAS to the varying communication topologies. In addition, tracking problems of heterogeneous and nonrepetitive MAS were studied in [13, 14]. Devasia [13] addressed the convergence of ILC for networked and heterogeneous MAS, where each agent has potentially different dynamics. And, the authors in [14] presented a novel ILC algorithm that did not rely on identical reference trajectories over the iteration domain. Besides, data-driven frameworks of ILC for MAS were proposed in $[15,16]$. Coincidentally, $\mathrm{Bu}$ et al. made a more in-depth study on data-driven ILC and solved the problem of data quantization and limited sensor 
output [17-19]. Bandwidth issues [20] and cyberattacks also increase the difficulty of achieving multiagent consistency control. In [21], a method of dynamic event-triggered scheduling was proposed to deal with the problem of limited bandwidth. In [22], distributed secure control and resourceaware secure control methods are introduced to ensure the security of multiagent networks.

However, an underlying condition of all the abovementioned results is that the signal transmission is reliable, which means that signals were not polluted when they are transmitted from one agent to another. But for MAS, all agents are usually located in different sites and communicate with others via wireless networks. Then, channel noise and data dropout always exist in channels of wireless networks and cause interference to the transmitted signals. Thus, reliable transmission is hard to be realized in actual networks when there are channel noise and data dropout in the wireless communication channel. This increases the design difficulty of iterative learning controllers. To solve this problem, many references give corresponding solutions on channel noise and data dropout. First of all, Kalman filter [23, 24] and adaptive methods [25-27] are general ways to eliminate the effect of channel noise on ILC. In addition, Shen and Qu [28] considered the fading channel with both multiplicative and additive randomness, and a decreasing gain sequence was applied in the design of the iterative learning controller. After that, in [29], the robustness of ILC for a class of mobile robot systems with channel noise was analyzed. Then, distributed iterative algorithms for consensus problems were considered for random switching networks with channel noise [30]. Also, data dropout is a common problem existing in wireless communication channels and developing on ILC for many years [31-34]. The convergence of ILC with successively dropped input data for a class of discrete-time systems over networks was discussed in [31]. Besides, Shen proposed an iterative learning method for linear stochastic systems under general data dropout environments [32] and then extended it to the data-driven approach [33].

Nevertheless, consensus problems of MAS with ILC in presence of channel noise or data dropout have not been fully investigated. These abovementioned references only consider the impact of channel noise or only the impact of data dropout. It is well known that channel noise and data dropout are unavoidable in wireless communication channels, and they simultaneously act on transmitted signals when the signals are transmitted from one agent to another. Channel noise leads to an inaccurate signal, and data dropout means that the signal is unable to be received by other agents. In other words, the signal cannot be received if data dropout exists; otherwise, the signal is received by other agents in an imprecise form. Then, these combined effects of channel noise and data dropout act on the iterative learning controller, causing the ILC algorithms to fail to converge. This indicates that the design complexity of the iterative learning controller is greatly increased when channel noise and data dropout coexist. Unfortunately, no literature considers channel noise and data dropout simultaneously for ILC of MAS, and ILC schemes proposed by existing references cannot solve this problem well. Therefore, our aim is to design an iterative learning controller to guarantee the convergence of ILC for MAS with channel noise and data dropout.

Motivated by this, in this paper, channel noise and data dropout are both taken into account for MAS. The mixing error caused by channel noise and data dropout will be introduced into the iterative learning controller and then make the outputs of MAS more difficult to reach the desired output. For dealing with this problem, an average-ILC method is proposed. As a result, rigorous theories demonstrate that the effects of both channel noise and data dropout on iterative learning controllers can be effectively eliminated by the proposed method.

The main contributions of this paper are highlighted as follows.

(1) As we all know, channel noise and data dropout are important factors affecting signal transmission. As far as we know, there is no paper to study the situation with both channel noise and data loss. Thus, unlike the aforementioned references, channel noise and data dropout are both considered for the consensus problem of MAS using ILC, which further promotes the design complexity of the iterative learning controller and then increases the difficulty of the consensus problem for MAS. In addition, the differences between channel noise and data dropout are described, and their combined effects on ILC for MAS are also depicted in this paper.

(2) For channel noise and data dropout introduced by wireless communication channels between neighboring agents, an average-iterative learning controller is designed to eliminate the mixing error caused by them. With the help of the series convergence property, a sufficient condition is proposed. Then, the perfect tracking can be obtained for MAS. In addition, by comparing the method proposed in this article with traditional methods, we verify the effectiveness of the method proposed in this article.

The remainder of this paper is organized as follows. Some preliminaries are made in Section 2, and the consensus problem of MAS with channel noise and data dropout is formulated in Section 3. To deal with this problem, an average-iterative learning scheme is proposed and the corresponding convergence is carefully analyzed in Section 4. The simulation results are shown in Section 5, and the effectiveness of the proposed method is illustrated. Finally, conclusions are given in Section 6.

Notions: $\varphi_{n}=\{1,2, \ldots, n\}$ represents $n$ agents, and $\mathbb{Z}_{N}=$ $\{0,1, \ldots, N\}$ contains all discrete time. 1 and 0 denote the column vector with appropriate dimensions whose elements are all ones and all zeros, respectively. $\mathbf{I}$ is an identity matrix with appropriate dimensions.

\section{Preliminaries}

2.1. Graph Theory of MAS. Based on algebraic graph theory, we can change the network topology into mathematics matrices. Let $\mathscr{G}=\{V, E\}$ be a directed graph consisting of $n$ 
agents, where $V=\left\{v_{j}: j \in \varphi_{n}\right\} \quad$ is $n$ agents and $E \subseteq\left\{\left(v_{i}, v_{j}\right): v_{i}, v_{j} \in V\right)$ is the edge set, and an edge $\left(v_{i}, v_{j}\right)$ represents a wireless channel where signals are transmitted from $v_{i}$ to $v_{j}$. Therefore, we can associate the adjacency weight of $\left(v_{i}, v_{j}\right)$ with $a_{j i}$. Define $a_{j i}=1$ when $\left(v_{i}, v_{j}\right) \in E$; otherwise, $a_{j i}=0$. Moreover, we assume that $a_{i i}=0$, that is,

$$
a_{j i}= \begin{cases}1, & \left(v_{i}, v_{j}\right) \in E, \\ 0, & \left(v_{i}, v_{j}\right) \notin E \text { or } j=i .\end{cases}
$$

A path in directed graph $\mathscr{G}$ is a finite sequence $\left(v_{j_{1}}, v_{j_{2}}, \ldots, v_{j_{l}}\right)$ and $\left(v_{j_{i}}, v_{j_{i+1}}\right) \in E, i=1,2, \ldots, l-1$. Define $\mathscr{A}=\left[a_{j i}\right] \in \mathbb{R}^{n \times n}$ is a nonnegative weighted adjacency matrix of directed graph $\mathscr{G}$. Define $D=\operatorname{diag}\left\{d_{1}, d_{2}, \ldots, d_{n}\right\}$ where $d_{j}=\sum_{i \in \mathcal{N}_{j}} a_{j i}$ is the sum of $j$ th row of the weighted adjacency matrix $\mathscr{A} \cdot \mathscr{N}_{j}=\left\{v_{i} \in V:\left(v_{i}, v_{j}\right) \in E\right\}$ represents the set of neighbors of agent $j$. The Laplacian matrix of $\mathscr{G}$ is defined as $L=D-\mathscr{A}$. If MAS has a virtual leader marked as $r$, then the network topology can be described as $\overline{\mathscr{G}}=\{\bar{E}, \mathscr{V} \cup\{r\}\}$, where $\bar{E} \subseteq\left\{\left(v_{i}, v_{j}\right): v_{i}, v_{j} \in \mathscr{V} \cup\{r\}\right\}$. Define $H=L+\overline{\mathscr{B}}$ where $\overline{\mathscr{B}}=\operatorname{diag}\left\{b_{1}, b_{2}, \ldots, b_{n}\right\}$ with $b_{j}=1$ if $j$ th agent can obtain information from the virtual leader $r$ directly; otherwise, $b_{j}=0$.

2.2. Channel Noise and Packet Loss of MAS. All agents of MAS are located in different positions, and the multiagent consistency is realized through mutual cooperation. This means each agent needs to send signals to others and receive signals from others via wireless networks. However, channel noise and data dropout in wireless networks can cause interference to transmitted signals. Then, the details of channel noise and data dropout are shown in Figure 1. In this figure, agents 1,2 , and 3 are neighboring agents of agent $j$. It is easy to obtain that outputs of neighboring agents (agents 1,2, and 3 ) are polluted before agent $j$ receives them.

For instance, agent 1 transmits its output $\eta(t)$ to agent $j$ via wireless networks. When the output is sent from agent 1 , it is $\eta(t)$, but when it is received by other agents, it is $\widehat{\eta}(t)=\mu(t)(\eta(t)+m(t))$, where $\eta(t)$ is the original signal, $m(t)$ is additive white Gaussian noise, and $\mu(t)$ is the randomness of data dropout which follows the Bernoulli distribution. Herein, there is no data dropout if $\mu(t)=1$; otherwise, $\mu(t)=0$. To better describe the randomness of these two variables, we assume that $\mu(t)$ and $m(t)$ satisfy the following statistics:

$$
\left\{\begin{array}{l}
x_{j, k}(t+1)=A x_{j, k}(t)+B u_{j, k}(t), \\
y_{j, k}(t)=C x_{j, k}(t),
\end{array}\right.
$$

where $x_{j}(t) \in \mathbb{R}, y_{j}(t) \in \mathbb{R}$, and $u_{j}(t) \in \mathbb{R}$ represent the state, output, and control input of $j$ th agent, respectively. $A$, $B$, and $C$ are system parameters.

For describing all agents of MAS (3), we denote $x_{k}(t)=\left[x_{1, k}(t), \ldots, x_{n, k}(t)\right]^{T}, \quad y_{k}(t)=\left[y_{1, k}(t), \ldots\right.$,

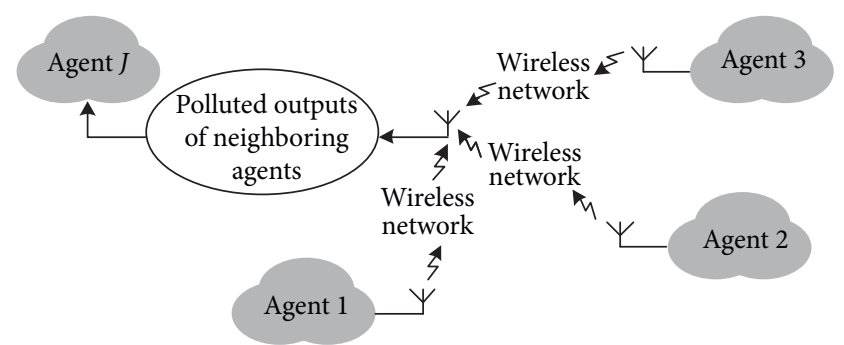

FIgURE 1: Channel noise and data dropout between agent $j$ and its neighboring agents.

$$
\begin{aligned}
\mathbb{E}[\mu(t)] & =\mu, \\
\mathbb{E}[m(t)] & =0, \\
\sigma_{m}^{2}=\mathbb{E}\left[m^{2}(t)\right] & =R_{m},
\end{aligned}
$$

where $0<\mu \leq 1$ represents the successful transfer rate and $R_{m}$ is the variance of channel noise $m(t)$.

The effects of both channel noise and data dropout on agent $j$ are shown in Figure 2. The controller obtains the mixing error $\widehat{\xi}_{j, k}(t)$, which contains the randomness of channel noise and data dropout. Therefore, the design complexity of the iterative learning controller increases. This means that we need a novel iterative learning scheme to eliminate these effects. For the ease of presentation, we consider the noise as additive white Gaussian noise. In follow-up studies, we will consider other more general noises.

Remark 1. It also assumes that $R_{m}$ and $\mu$ are known to us because the statistics of both channel noise and data dropout rely on the actual environment. Therefore, in order to obtain the probability of data dropout $\mu$, we can get the frequency of data dropout through multiple trials. When the number of trials is large enough, the frequency of data dropout is equal to the probability of data dropout. This means the probability of data dropout can be estimated in advance. Besides, $\mu(t)$ is independent of $m(t)$, that is, $\mathbb{E}[\mu(t) m(t)]=\mathbb{E}[\mu(t)] \mathbb{E}[m(t)]$.

\section{Problem Formulation}

Consider MAS with $n$ agents and the $j$ th one is governed by the following discrete-time dynamics:

$\forall t \in \mathbb{Z}_{N}, j \in \varphi_{n}$, and $k=0,1, \ldots$,

$\left.y_{n, k}(t)\right]^{T}$, and $u_{k}(t)=\left[u_{1, k}(t), \ldots, u_{n, k}(t)\right]^{T}$. Then, we can rewrite (3) as

$$
\left\{\begin{array}{l}
x_{k}(t+1)=A x_{k}(t)+B u_{k}(t) \\
y_{k}(t)=C x_{k}(t)
\end{array}\right.
$$




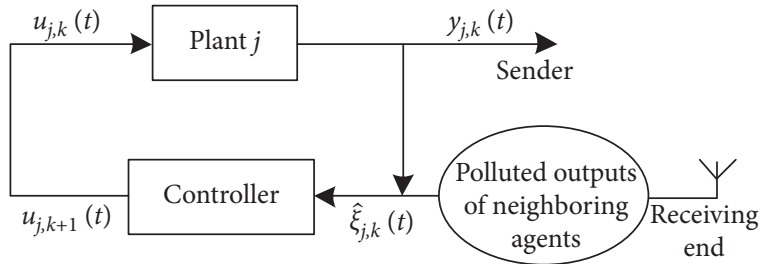

FIgURE 2: Iterative process within agent $j$.

where $\mathbf{A}=\operatorname{diag}\{A, A, \ldots, A\} \in \mathbb{R}^{n \times n}, \mathbf{B}=\operatorname{diag}\{B, B, \ldots, B\}$ $\in \mathbb{R}^{n \times n}$, and $\mathbf{C}=\operatorname{diag}\{C, C, \ldots, C\} \in \mathbb{R}^{n \times n}$.

For a given virtual leader $r$, its output trajectory $y_{r}(t) \in \mathbb{R}$ is the desired trajectory for MAS. In this paper, desired control input $u_{r}(t) \in \mathbb{R}$ satisfies

$$
\left\{\begin{array}{l}
x_{r}(t+1)=A x_{r}(t)+B u_{r}(t), \\
y_{r}(t)=C x_{r}(t),
\end{array}\right.
$$

where $x_{r}(t) \in \mathbb{R}$ is the desired state.

Based on the descriptions of channel noise and data dropout in Section 2, we learn that both channel noise and data dropout existing in wireless networks pollute the transmitted signals and then increase the uncertainty of MAS. Take agent $j$ for example, the output that is received from agent $i$ can be described as

$$
\tilde{y}_{i, k}(t)=\mu_{i, k}(t)\left[y_{i, k}(t)+m_{i j, k}(t)\right] .
$$

For the convenience of the following analysis, $\mu^{-1}$ is applied in (6). Then, we have

$$
\begin{aligned}
\hat{y}_{i, k}(t) & =\mu^{-1} \tilde{y}_{i, k}(t), \\
& =\mu^{-1} \mu_{i, k}(t)\left[y_{i, k}(t)+m_{i j, k}(t)\right], \\
& =y_{i, k}(t)-\left(1-\mu^{-1} \mu_{i j, k}(t)\right) y_{i, k}(t)+\mu^{-1} \mu_{i j, k}(t) m_{i j, k}(t), \\
& =y_{i, k}(t)-\bar{\mu}_{i j, k}(t) y_{i, k}(t)+\bar{m}_{i j, k}(t),
\end{aligned}
$$

where $\quad \bar{\mu}_{i j, k}(t)=1-\mu^{-1} \mu_{i, k}(t) \quad$ and $\quad \bar{m}_{i j, k}(t)=\mu^{-1} \mu_{i j, k}$ $(t) m_{i j, k}(t) . \mu$ is described in (2).

Remark 2. $y_{i, k}(t)$ is the output of agent $i$ and $\hat{y}_{i, k}(t)$ is the signal received by the neighboring agents of agent $i$. It is obvious that $\hat{y}_{i, k}(t)$ is affected by channel noise and data dropout in wireless channels, and then, $\mathbb{E}\left[\bar{\mu}_{i j, k}(t)\right]=0$. Corresponding to the actual process, the first step of (7) means that agent $j$ receives the polluted output of agent $i$ and then multiplies the factor $\mu^{-1}$, which is known to us in advance.

We consider that the topology of MAS (3) has a distributed structure, which means that part of agents can obtain signals from the virtual leader $r$ only. Therefore, local error for agent $j$ at time $t$ and iteration $k$ can be expressed as

$$
\begin{aligned}
\widehat{\xi}_{j, k}(t) & =\sum_{i \in \mathcal{N}_{j}} a_{j i}\left(\hat{y}_{i, k}(t)-y_{j, k}(t)\right)+b_{j}\left(y_{r}(t)-y_{j, k}(t)\right), \\
& =\sum_{i \in \mathcal{N}_{j}} a_{j i}\left(y_{i, k}(t)-y_{j, k}(t)\right)+b_{j}\left(y_{r}(t)-y_{j, k}(t)\right)+\sum_{i \in \mathcal{N}_{j}} a_{j i}\left(-\bar{\mu}_{i j, k}(t) y_{i, k}(t)\right)+\bar{m}_{j, k}(t),
\end{aligned}
$$

where $\bar{m}_{j, k}(t)=\sum_{i \in \mathcal{N}_{j}} a_{j i} \bar{m}_{i j, k}(t)$

Define $\bar{m}_{k}(t)=\left[\bar{m}_{1, k}(t), \bar{m}_{2, k}(t), \ldots, \bar{m}_{n, k}(t)\right]^{T}$ and $\overline{\mathscr{A}}_{k}(t)=\left[\bar{a}_{j i, k}(t)\right]$ where $\bar{a}_{j i}(t)=a_{j i} \bar{\mu}_{i j, k}(t)$ is the element of $j$ th row and $i$ th column in matrix $\mathscr{A}_{k}(t)$. Then, the compact form of (8) is

$$
\begin{aligned}
\widehat{\xi}_{k}(t)= & H\left(1_{n} y_{r}(t)-y_{k}(t)\right)+\overline{\mathscr{A}}_{k}(t)\left(1_{n} y_{r}(t)-y_{k}(t)\right) \\
& -\overline{\mathscr{A}}_{k}(t) 1_{n} y_{r}(t)+\bar{m}_{k}(t) .
\end{aligned}
$$

For MAS (3), the tracking error at time $t$ and iteration $k$ can be described as follows:

$$
e_{k}(t)=1 y_{r}(t)-y_{k}(t)
$$

Then, (9) can be rewritten as

$$
\widehat{\xi}_{k}(t)=H e_{k}(t)+\overline{\mathscr{A}}_{k}(t) e_{k}(t)-\overline{\mathscr{A}}_{k}(t) 1 y_{r}(t)+\bar{m}_{k}(t) \text {. }
$$

Define $\quad \mathbf{y}_{r}=\left[1^{T} y_{r}(1), 1^{T} y_{r}(2), \ldots, 1^{T} y_{r}(N)\right]^{T}, \quad \mathbf{y}_{k}=$ $\left[y_{k}^{T}(1), y_{k}^{T}(2), \ldots, y_{k}^{T}(N)\right]^{T}, \quad$ and $\mathbf{e}_{k}=\left[e_{k}^{T}(1), e_{k}^{T}(1)\right.$, $\left.\ldots, e_{k}^{T}(N)\right]^{T}$. Then, the compact form of (10) is

$$
\mathbf{e}_{k}=\mathbf{y}_{\mathbf{r}}-\mathbf{y}_{k} \text {. }
$$

Then, considering all the time, we can just go on and derive the following equations from (9):

$$
\begin{aligned}
\widehat{\boldsymbol{\xi}}_{k} & =\mathrm{H}\left(\mathbf{y}_{\mathbf{r}}-\mathbf{y}_{k}\right)+\overline{\mathscr{A}}_{k}\left(\mathbf{y}_{\mathbf{r}}-\mathbf{y}_{k}\right)-\overline{\mathscr{A}}_{k} \mathbf{y}_{r}+\overline{\mathbf{m}}_{k}, \\
& =\mathrm{He}_{k}+\overline{\mathscr{A}}_{k} \mathbf{e}_{k}-\overline{\mathscr{A}}_{k} \mathbf{y}_{r}+\overline{\mathbf{m}}_{k},
\end{aligned}
$$

where $\hat{\xi}_{k}=\left[\xi_{k_{T}}^{T}(1), \xi_{k}^{T}(2), \ldots, \xi_{k}^{T}(N)\right]^{T}, \overline{\mathbf{m}}_{k}=\left[\bar{m}_{k}^{T}(1), \bar{m}_{k}^{T}(2\right.$ )$\left., \ldots, \bar{m}_{k}^{T}(N)\right]^{T}, \quad \mathrm{H}=\mathrm{H} \otimes I_{N}, \quad$ and $\quad \overline{\mathscr{A}}_{k}=\operatorname{diag}\left\{\overline{\mathscr{A}}_{k}(1)\right.$, $\left.\overline{\mathscr{A}}_{k}(2), \ldots, \overline{\mathscr{A}}_{k}(N)\right\}$ for simplicity.

The objective in this paper is to ensure that the following convergence principle holds:

$$
\lim _{k \longrightarrow \infty} \mathbb{E}\left[\mathbf{e}_{k} \mathbf{e}_{k}^{T}\right]=0
$$


Remark 3. It is clear that $\widehat{\xi}_{k}$ is the local error polluted by channel noise and data dropout, while $\mathbf{e}_{k}$ is the tracking error which represents the error between the outputs of all agents and desired output. In particular, channel noise and data dropout only have effect on the outputs that need to be transmitted, but for the agent itself, its output is not polluted by channel noise and data dropout. Thus, what we should do is to design the iterative learning scheme to eliminate the combined effects of channel noise and data dropout on the iterative learning controller and then to guarantee that all outputs of agents reach the desired output, such as (14).

Assumption 1. The virtual leader $r$ is globally reachable in directed graph $\overline{\mathscr{G}}$.

Assumption 2. For all agents $i \in \varphi_{n}$ and iteration $k=0,1, \ldots$, the initial-time state satisfies

$$
x_{i, k}(0)=x_{r}(0), \quad \forall i \in \varphi_{n} \text { and } k=0,1, \ldots
$$

Assumption 3. For all agents $i \in \varphi_{n}$, time $t \in \mathbb{Z}_{N}$ and iteration $k=0,1, \cdots$, and the channel noise $m_{i, k}(t)$ is bounded.

Remark 4. Assumption 2 is a common one in research studies on ILC $[25,29]$. If the assumption cannot be satisfied, some methods can be applied to deal with the problem of the initial state, such as adaptive ILC with the neural network [35]. Thereby, for our future work, one of our objectives is to consider the initial state into the framework of ILC for MAS with channel noise and data dropout. Assumption 3 is also a general condition for the controller design $[25,28]$. The channel noise is bounded but the bound is unknown. This is consistent with the actual situation.

\section{Design of Average-Iterative Learning Controller}

Channel noise and data dropout widely exist in the process of wireless signal transmission, which increases the design difficulty of the iterative learning controller. Hence, the research of channel noise and data dropout on ILC is undoubtedly important. In this section, an iterative learning controller is designed for MAS. An average-iterative learning scheme is utilized to deal with the combined effects of channel noise and data dropout on iterative learning controllers.

For realizing objective (14), an average-iterative learning scheme of agent $j$ at time $t$ and iteration $k$ is proposed as follows:

$$
u_{j, k+1}(t)=\frac{1}{k+1} \sum_{q=0}^{k}\left[u_{j, q}(t)+\Gamma_{j}(t) \widehat{\xi}_{j, q}(t+1)\right] .
$$

According to the definition of $u_{k}(t)$ in (4), we have

$$
u_{k+1}(t)=\frac{1}{k+1} \sum_{q=0}^{k}\left[u_{q}(t)+\Gamma(t) \widehat{\xi}_{q}(t+1)\right]
$$

where $\Gamma(t)=\operatorname{diag}\left\{\Gamma_{1}(t), \Gamma_{2}(t), \ldots, \Gamma_{n}(t)\right\}$.

Define $\quad \mathbf{u}_{k}=\left[u_{k}(0), u_{k}(1), \ldots, u_{k}(N-1)\right] \quad$ and $\Gamma=\operatorname{diag}\{\Gamma(0), \Gamma(1), \ldots, \Gamma(N-1)\}$, and we can obtain the compact form of (17):

$$
\mathbf{u}_{k+1}=\frac{1}{k+1} \sum_{q=0}^{k}\left[\mathbf{u}_{q}+\Gamma \widehat{\xi}_{q}\right] .
$$

For MAS (3), we define the desired input $\mathbf{u}_{r}=\left[1_{n}^{T} u_{r}(0), 1_{n}^{T} u_{r}(1), \ldots, 1_{n}^{T} u_{r}(N-1)\right]^{T}$ and input error $\Delta \mathbf{u}_{k}=\mathbf{u}_{r}-\mathbf{u}_{k}$. Substituting (13) into (18), the compact form of our learning scheme can be converted as follows:

$$
\mathbf{u}_{k+1}=\frac{1}{k+1} \sum_{q=0}^{k}\left\{\mathbf{u}_{q}+\Gamma H \mathbf{e}_{q}+\Gamma \overline{\mathscr{A}}_{q} \mathbf{e}_{q}-\Gamma \overline{\mathscr{A}}_{q} \mathbf{y}_{r}+\Gamma \bar{m}_{q}\right\},
$$

$\Delta \mathbf{u}_{k+1}=\frac{1}{k+1} \sum_{q=0}^{k}\left\{\Delta \mathbf{u}_{q}-\Gamma \mathrm{H} \mathbf{e}_{q}-\Gamma \overline{\mathscr{A}}_{q} \mathbf{e}_{q}+\Gamma \overline{\mathscr{A}}_{q} \mathbf{y}_{r}-\Gamma \bar{m}_{q}\right\}$.

According to the expression of (20), it shows the relation of $\Delta \mathbf{u}_{k+1}$ and $\Delta \mathbf{u}_{k}$. However, the relation of $\Delta \mathbf{u}_{k}$ and $\mathbf{e}_{k}$ is not clear, and it plays an important role in our next analysis. Then, we have the following derivation. Based on (10), we can derive that

$$
\begin{aligned}
e_{k}(t) & =1 y_{r}(t)-y_{k}(t), \\
& =\mathbf{C}\left(1 x_{r}(t)-x_{k}(t)\right), \\
& =\mathbf{C}\left(\mathbf{A} \Delta x_{k}(t-1)+\mathbf{B} \Delta u_{k}(t-1)\right), \\
& =\mathbf{C}\left(\mathbf{A}^{t} \Delta x_{k}(0)+\sum_{i=0}^{t-1} \mathbf{C} \mathbf{A}^{i} \mathbf{B} \Delta u_{k}(t-i-1)\right), \\
& =\sum_{i=0}^{t-1} \mathbf{C}\left(\mathbf{A}^{i} \mathbf{B} \Delta u_{k}(t-i-1)\right),
\end{aligned}
$$

where Assumption 2 is applied in the fourth step.

Then, the compact form of tracking error (12) can be rewritten as follows:

$$
\mathbf{e}_{k}=\mathbf{M} \Delta \mathbf{u}_{k}
$$

where

$$
\mathbf{M}=\left[\begin{array}{cccc}
\mathbf{C B} & \mathbf{0} & \ldots & \mathbf{0} \\
\mathbf{C A B} & \mathbf{C B} & \ldots & 0 \\
\vdots & \vdots & \ddots & \ddots \\
\mathbf{C A}^{N-1} \mathbf{B} & \mathbf{C A}^{N-2} \mathbf{B} & \ldots & \mathbf{C B}
\end{array}\right]
$$

The relation of $\Delta \mathbf{u}_{k}$ and $\mathbf{e}_{k}$ is deduced in (22). Then, we take (22) into (20), 


$$
\begin{aligned}
\Delta \mathbf{u}_{k+1}= & \frac{1}{k+1} \sum_{q=0}^{k}\left[(\mathbf{I}-\Gamma \mathrm{H}) \Delta \mathbf{u}_{q}-\Gamma \overline{\mathscr{A}}_{q} \mathbf{M} \Delta \mathbf{u}_{q}+\Gamma \overline{\mathscr{A}}_{q} \mathbf{y}_{r}-\Gamma \bar{m}_{q}\right] \\
= & \frac{1}{k+1}\left[(\mathbf{I}-\Gamma \mathrm{M}) \Delta \mathbf{u}_{k}-\Gamma \overline{\mathscr{A}}_{k} \mathbf{M} \Delta \mathbf{u}_{k}+\Gamma \overline{\mathscr{A}}_{k} \mathbf{y}_{r}-\Gamma \bar{m}_{k}\right] \\
& +\frac{k}{k+1} \times \frac{1}{k} \sum_{q=0}^{k-1}\left[(\mathbf{I}-\Gamma \mathrm{H}) \Delta \mathbf{u}_{q}-\Gamma \overline{\mathscr{A}}_{q} \mathbf{M} \Delta \mathbf{u}_{q}+\Gamma \overline{\mathscr{A}}_{q} \mathbf{y}_{r}-\Gamma \bar{m}_{q}\right] \\
= & \frac{1}{k+1}\left[(\mathbf{I}-\Gamma \mathrm{M}) \Delta \mathbf{u}_{k}-\Gamma \overline{\mathscr{A}}_{k} \mathbf{M} \Delta \mathbf{u}_{k}+\Gamma \overline{\mathscr{A}}_{k} \mathbf{y}_{r}-\Gamma \bar{m}_{k}\right]+\frac{k}{k+1} \Delta \mathbf{u}_{k}, \\
= & \mathrm{P}_{k} \Delta \mathbf{u}_{k}-\frac{1}{k+1} \Gamma \overline{\mathscr{A}}_{k} \mathbf{M} \Delta \mathbf{u}_{k}+\frac{1}{k+1} \Gamma \overline{\mathscr{A}}_{k} \mathbf{y}_{r}-\frac{1}{k+1} \Gamma \bar{m}_{k},
\end{aligned}
$$

where $\mathrm{P}_{k}=\mathbf{I}-(1 /(k+1)) \Gamma \mathrm{H} \mathbf{M}$.

Benefitting from above derivations, we can obtain the following theorem and corollary. For the ease of representation, we define $\mathrm{V}_{k}=\left(\Delta \mathbf{u}_{k}\right)\left(\Delta \mathbf{u}_{k}\right)^{T}$.

Theorem 1. Let us consider MAS (3) with channel noise and data dropout, and Assumptions 1-3 hold. Average-iterative learning scheme (16) is applied. If there exists gain matrix $\Gamma$ satisfying

$$
\|\mathbf{I}-\Gamma \mathrm{H} \mathbf{M}\|=\rho<1,
$$

Proof. From (24) and definition of $\mathrm{V}_{k}$, we have following derivations:

$$
\begin{aligned}
\mathrm{V}_{k+1}= & \left(\Delta \mathbf{u}_{k+1}\right)\left(\Delta \mathbf{u}_{k+1}\right)^{T}, \\
= & \mathrm{P}_{k} \mathrm{~V}_{k} \mathbf{P}_{k}^{T}+\frac{1}{(k+1)^{2}}\left(\Gamma \overline{\mathscr{A}}_{k} \mathbf{M}\right) \mathrm{V}_{k}\left(\Gamma \overline{\mathscr{A}}_{k} \mathbf{M}\right)^{T}+\frac{1}{(k+1)^{2}}\left(\Gamma \overline{\mathscr{A}}_{k} \mathbf{y}_{r}\right)\left(\Gamma \overline{\mathscr{A}}_{k} \mathbf{y}_{r}\right)^{T}+\frac{1}{(k+1)^{2}} \Gamma \bar{m}_{k} \bar{m}_{k}^{T} \Gamma^{T} \\
& -\frac{1}{k+1} \mathrm{P}_{k} \mathrm{~V}_{k}\left(\Gamma \overline{\mathscr{A}}_{k} \mathbf{M}\right)^{T}-\frac{1}{k+1}\left(\Gamma \overline{\mathscr{A}}_{k} \mathbf{M}\right) \mathrm{V}_{k} \mathbf{P}_{k}^{T}+\frac{1}{k+1} \mathrm{P}_{k} \Delta \mathbf{u}_{k}\left(\Gamma \overline{\mathscr{A}}_{k} \mathbf{y}_{r}\right)^{T}+\frac{1}{k+1}\left(\Gamma \overline{\mathscr{A}}_{k} \mathbf{y}_{r}\right)\left(\Delta \mathbf{u}_{k}\right)^{T} \mathbf{P}_{k}^{T}-\frac{1}{k+1} \mathrm{P}_{k} \Delta \mathbf{u}_{k} \overline{\mathbf{m}}_{k}^{T} \Gamma^{T} \\
& -\frac{1}{k+1} \Gamma \bar{m}_{k}\left(\Delta \mathbf{u}_{k}\right)^{T} \mathbf{P}_{k}^{T}-\frac{1}{(k+1)^{2}} \Gamma \overline{\mathscr{A}}_{k} \mathbf{M} \Delta \mathbf{u}_{k}\left(\Gamma \overline{\mathscr{A}}_{k} \mathbf{y}_{r}\right)^{T}-\frac{1}{(k+1)^{2}}\left(\Gamma \overline{\mathscr{A}}_{k} \mathbf{y}_{r}\right)\left(\Delta \mathbf{u}_{k}\right)^{T}\left(\Gamma \overline{\mathscr{A}}_{k} \mathbf{M}\right)^{T} \\
& +\frac{1}{(k+1)^{2}} \Gamma \overline{\mathscr{A}}_{k} \mathbf{M} \Delta \mathbf{u}_{k} \bar{m}_{k}^{T} \Gamma^{T}+\frac{1}{(k+1)^{2}} \Gamma \bar{m}_{k}\left(\Delta \mathbf{u}_{k}\right)^{T}\left(\Gamma \overline{\mathscr{A}}_{k} \mathbf{M}\right)^{T}-\frac{1}{(k+1)^{2}}\left(\Gamma \overline{\mathscr{A}}_{k} \mathbf{y}_{r}\right)^{\bar{m}_{k}^{T}} \Gamma^{T}-\frac{1}{(k+1)^{2}} \Gamma \bar{m}_{k}\left(\Gamma \overline{\mathscr{A}}_{k} \mathbf{y}_{r}\right)^{T} .
\end{aligned}
$$

Noting that $\mathbb{E}\left[\bar{m}_{k}\right]=0$ and $\mathbb{E}\left[\overline{\mathscr{A}}_{k}\right]=0$. Besides, $\bar{m}_{k}$ and $\overline{\mathscr{A}}_{k}$ are mutually independent. Thus, we take mathematical expectations on both sides of (26):

$$
\begin{aligned}
\mathbb{E}\left[\mathrm{V}_{k+1}\right]= & \mathrm{P}_{k} \mathbb{E}\left[\mathrm{V}_{k}\right] \mathbf{P}_{k}^{T}+\frac{1}{(k+1)^{2}} \mathbb{E}\left[\left(\Gamma \overline{\mathscr{A}}_{k} \mathbf{M}\right) \mathbb{E}\left[\mathrm{V}_{k}\right]\left(\Gamma \overline{\mathscr{A}}_{k} \mathbf{M}\right)^{T}\right] \\
& +\frac{1}{(k+1)^{2}} \mathbb{E}\left[\left(\Gamma \overline{\mathscr{A}}_{k} \mathbf{y}_{r}\right)\left(\Gamma \overline{\mathscr{A}}_{k} \mathbf{y}_{r}\right)^{T}\right]+\frac{1}{(k+1)^{2}} \Gamma \mathbb{E}\left[\bar{m}_{k} \bar{m}_{k}^{T}\right] \Gamma^{T} \\
& -\frac{1}{(k+1)^{2}} \mathbb{E}\left[\Gamma \overline{\mathscr{A}}_{k} \mathbf{M} \mathbb{E}\left[\Delta \mathbf{u}_{k}\right]\left(\Gamma \overline{\mathscr{A}}_{k} \mathbf{y}_{r}\right)^{T}\right]-\frac{1}{(k+1)^{2}} \mathbb{E}\left[\left(\Gamma \overline{\mathscr{A}}_{k} \mathbf{y}_{r}\right) \mathbb{E}\left[\left(\Delta \mathbf{u}_{k}\right)^{T}\right]\left(\Gamma \overline{\mathscr{A}}_{k} \mathbf{M}\right)^{T}\right] .
\end{aligned}
$$


Norm on both sides of (27):

$$
\begin{aligned}
\left\|\mathbb{E}\left[\mathrm{V}_{k+1}\right]\right\| \leq & \left\|\mathrm{P}_{k}\right\|^{2}\left\|\mathbb{E}\left[\mathrm{V}_{k}\right]\right\|+\frac{c_{1}}{(k+1)^{2}}\left\|\mathbb{E}\left[\mathrm{V}_{k}\right]\right\| \\
& +\frac{c_{2}}{(k+1)^{2}}+\frac{c_{3}}{(k+1)^{2}}\left\|\mathbb{E}\left[\Delta \mathbf{u}_{k}\right]\right\|,
\end{aligned}
$$

where $c_{1}, c_{2}$, and $c_{3}$ are suitable values. We can obtain from (24) that

$$
\begin{aligned}
\left\|\mathbb{E}\left[\Delta \mathbf{u}_{k+1}\right]\right\| & \leq\left\|\mathrm{P}_{k}\right\|\left\|\mathbb{E}\left[\Delta \mathbf{u}_{k}\right]\right\|, \\
& \leq \prod_{q=0}^{k}\left\|\mathrm{P}_{q}\right\|\left\|\mathbb{E}\left[\Delta \mathbf{u}_{0}\right]\right\| .
\end{aligned}
$$

It is worth noting that

$$
\begin{aligned}
\left\|\mathrm{P}_{k}\right\| & =\left\|\mathbf{I}-\frac{1}{k+1} \mathbf{I}+\frac{1}{k+1} \mathbf{I}-\frac{1}{k+1} \Gamma \mathbf{H} \mathbf{M}\right\|, \\
& \leq 1-\frac{1}{k+1}+\frac{1}{k+1} \| \mathbf{I}-\text { ГН } \mathbf{M} \|, \\
& \leq 1-\frac{1-\rho}{k+1}<1,
\end{aligned}
$$

where $\rho<1$. Thus, we can obtain from (29) that

$$
\left\|\mathbb{E}\left[\Delta \mathbf{u}_{k+1}\right]\right\| \leq\left\|\mathbb{E}\left[\Delta \mathbf{u}_{0}\right]\right\|,
$$

where $\left\|\mathbb{E}\left[\Delta \mathbf{u}_{0}\right]\right\|$ is the value that we can set. Formula (31) means $\left\|\mathbb{E}\left[\Delta \mathbf{u}_{k}\right]\right\|$ is bounded, and we can derive from (28) that

$$
\left\|\mathbb{E}\left[\mathrm{V}_{k+1}\right]\right\| \leq\left(1-\frac{b_{\rho}}{k+1}+\frac{c_{1}}{(k+1)^{2}}\right)\left\|\mathbb{E}\left[\mathrm{V}_{k}\right]\right\|+\frac{c_{4}}{(k+1)^{2}},
$$

where $b_{\rho}=1-\rho<1$ and $c_{4}$ is a suitable value.

Obviously, there is an iteration number $k_{0}$ so that when $k>k_{0}$, we have

$$
1-\frac{b_{\rho}}{k+1}+\frac{c_{1}}{(k+1)^{2}}<1 .
$$

Then, we can derive from (32) that

$$
\begin{aligned}
\left\|\mathbb{E}\left[\mathrm{V}_{k+1}\right]\right\| & \leq\left\|\mathbb{E}\left[\mathrm{V}_{k}\right]\right\|+\frac{c_{4}}{(k+1)^{2}}, \\
& \leq\left\|\mathbb{E}\left[\mathrm{V}_{0}\right]\right\|+c_{4} \sum_{q=0}^{k} \frac{1}{(q+1)^{2}}<\infty .
\end{aligned}
$$

It means $\left\|\mathbb{E}\left[\mathrm{V}_{k+1}\right]\right\|$ is bounded. Thus, we can derive from (32) again:

$$
\begin{aligned}
\left\|\mathbb{E}\left[\mathrm{V}_{k+1}\right]\right\| & \leq\left(1-\frac{b_{\rho}}{k+1}\right)\left\|\mathbb{E}\left[\mathrm{V}_{k}\right]\right\|+\frac{c_{5}}{(k+1)^{2}}, \\
& =\left\|\mathbb{E}\left[\mathrm{V}_{k}\right]\right\|-\frac{1}{k+1}\left(b_{\rho}\left\|\mathbb{E}\left[\mathrm{V}_{k}\right]\right\|-\frac{c_{5}}{k+1}\right), \\
& =\left\|\mathbb{E}\left[\mathrm{V}_{k}\right]\right\|-\frac{b_{\rho}}{k+1}\left(\left\|\mathbb{E}\left[\mathrm{V}_{k}\right]\right\|-\frac{c_{5}}{b_{\rho}(k+1)}\right),
\end{aligned}
$$

where $c_{5}$ is a suitable value.

It seemed that if $\left\|\mathbb{E}\left[\mathrm{V}_{k}\right]\right\|$ cannot converge to zero, we have $\left\|\mathbb{E}\left[\mathrm{V}_{k}\right]\right\| \geq c_{6}$, where $c_{6}$ is a suitable value. Thus, we can derive from (35) that

$$
\begin{aligned}
\left\|\mathbb{E}\left[V_{k+1}\right]\right\| & \leq\left\|\mathbb{E}\left[\mathrm{V}_{k}\right]\right\|-\frac{b_{\rho} c_{6}}{k+1}, \\
& \leq\left\|\mathbb{E}\left[V_{0}\right]\right\|-\sum_{q=0}^{k} \frac{b_{\rho} c_{6}}{q+1} .
\end{aligned}
$$

It is worth noting that $\sum_{q=0}^{k}\left(\left(b_{\rho} c_{6}\right) /(q+1)\right) \longrightarrow \infty$ when $k \longrightarrow \infty$. Then, we have $\left\|\mathbb{E}\left[\mathrm{V}_{k+1}\right]\right\|<0$. Obviously, it is impossible. Thus, we can derive that

$$
\lim _{k \rightarrow \infty}\left\|\mathbb{E}\left[\mathrm{V}_{k}\right]\right\|=0
$$

This completes the proof.

Corollary 1. Let consider MAS (3) with channel noise and data dropout, and Assumptions 1-3 hold. Average-iterative learning scheme (16) is applied. Then, the covariance matrix of tracking error $\mathbf{e}_{k}$ asymptotically converges to zero if condition (25) holds, that is, $\lim _{k \longrightarrow \infty}\left\|\mathbb{E}\left[\mathbf{e}_{k} \mathbf{e}_{k}^{T}\right]\right\|=0$.

Proof. According to the relation of input error $\Delta \mathbf{u}_{k}$ and global output error $\mathbf{e}_{k}$, we can derive from (22) that

$$
\left\|\mathbb{E}\left[\mathbf{e}_{k} \mathbf{e}_{k}^{T}\right]\right\|=\|\mathbf{M}\|^{2}\left\|\mathbb{E}\left[\mathrm{V}_{k}\right]\right\|,
$$

where $\mathbf{M}$ is a constant matrix.

According to Theorem 1, we can derive that (37) holds if condition (25) holds. Then, we have

$$
\lim _{k \longrightarrow \infty}\left\|\mathbb{E}\left[\mathbf{e}_{k} \mathbf{e}_{k}^{T}\right]\right\|=0 .
$$

This completes the proof.

Remark 5. Theorem 1 illustrates that $\left\|\mathbb{E}\left[V_{k}\right]\right\|$ converts to zero in the mean-square sense with the help of average-iterative learning scheme (16). Obviously, the key to the design of the learning scheme is to require the gain matrix $\Gamma$ to satisfy sufficient condition (25). Corollary 1 derives that the covariance matrix of tracking error $\mathbf{e}_{k}$ converges to zero if $\left\|\mathbb{E}\left[V_{k}\right]\right\|$ converges to zero. It shows that the effects of both channel noise and data dropout on iterative learning controllers can be effectively eliminated by proposed scheme (16), and then, a perfect tracking can be guaranteed for MAS (3).

\section{Simulation Results}

For illustration and verification, we consider discrete-time MAS (3) with four agents and one virtual leader $r$. The system parameter metrics are shown in (40):

$$
\begin{aligned}
& A=0.3, \\
& B=C=1 .
\end{aligned}
$$




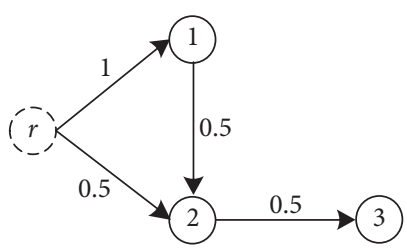

FIgURE 3: Topology of MAS with four agents and a virtual leader.

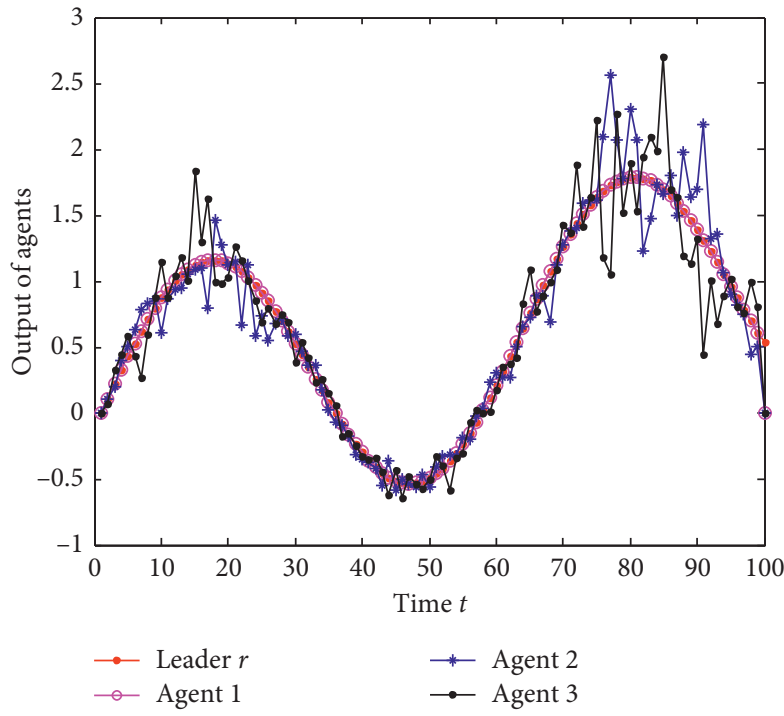

(a)

Figure 4: The outputs of MAS for $k=100$. (a) Benchmark method. (b) Proposed method.

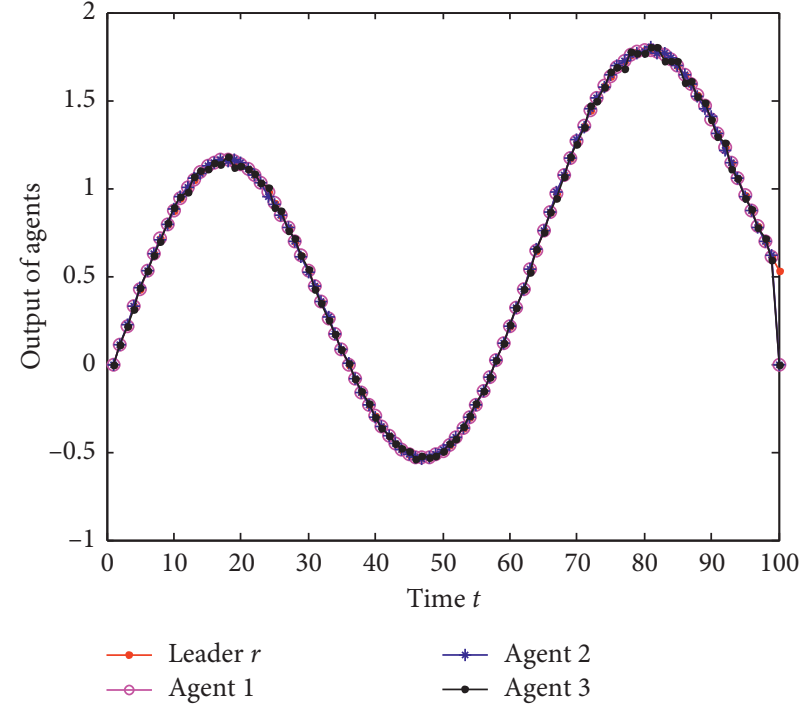

(b)
Let the desired output is $y_{r}(t)=(t / 100)+\sin (t / 10)$ with $t \in[0,100]$. The directed graph of MAS is shown in Figure 3. Then, the weighted adjacent matrix

$$
H=\left[\begin{array}{ccc}
1 & 0 & 0 \\
-0.5 & 1 & 0 \\
0 & -0.5 & 0.5
\end{array}\right] .
$$

Average-iterative learning scheme (16) is applied. For all agents, set the state at initial time $x_{j, k}(0)=0$. The gain matrix is set to be $\Gamma_{j}(t)=0.6$.

To show the effectiveness of the proposed method, a benchmark method is introduced, such as $u_{j, k+1}(t)=u_{j, k}(t)+\Gamma_{j}(t) \widehat{\xi}_{j, k}(t+1)$. The only difference between the proposed method and benchmark one is the design of the iterative learning scheme.

Figure 4 shows the outputs of the proposed method and the benchmark one, respectively, when the variance of channel noise is $\sigma_{m}^{2}=0.1$, and the successful transfer rate is $\mu=0.9$. From an intuitive point of view, the effect of outputs of MAS with the proposed method is better than the benchmark one.

To show the effectiveness of the proposed method more intuitively, we compare the convergence of the covariance matrix under the proposed method and benchmark method, such as Figure 5. The setting of channel noise and data dropout is the same as Figure 4 . Obviously, the result illustrates that the benchmark method cannot guarantee that the covariance matrix of tracking error converges to zero, whereas the proposed method does.

In this paper, channel noise and data dropout are both considered, and the effects of these two factors on the convergence speed of the covariance matrix of tracking error need to be analyzed when the proposed method is applied.

Figure 6 shows the effects of different variances of channel noise on convergence speed of the covariance matrix of tracking error. In this figure, the successful transfer rate is $\mu=0.95$, and the variances of channel noise are $\sigma_{m}^{2}=0, \sigma_{m}^{2}=0.1$, and $\sigma_{m}^{2}=0.2$. Then, the result shows that variances of channel noise do affect the convergence speed of tracking error, which still converges to zero. Besides, the smaller the variances, the faster the convergence speed.

The effect of different successful transfer rates on the convergence speed of the covariance matrix of tracking error is depicted in Figure 7. Similarly, the variance of the channel noise is $\sigma_{m}^{2}=0.1$, and the successful transfer rates are $\mu=1$, $\mu=0.9$, and $\mu=0.8$. Then, the result of Figure 7 shows that the successful transfer rate affects the convergence speed of 


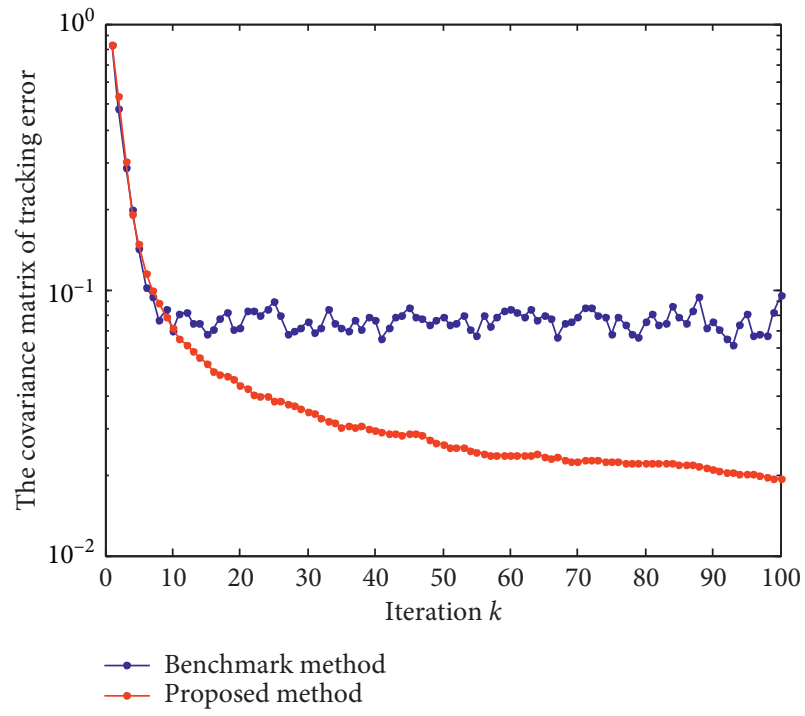

Figure 5: Convergence of the covariance matrix of tracking error with the proposed method or benchmark method.

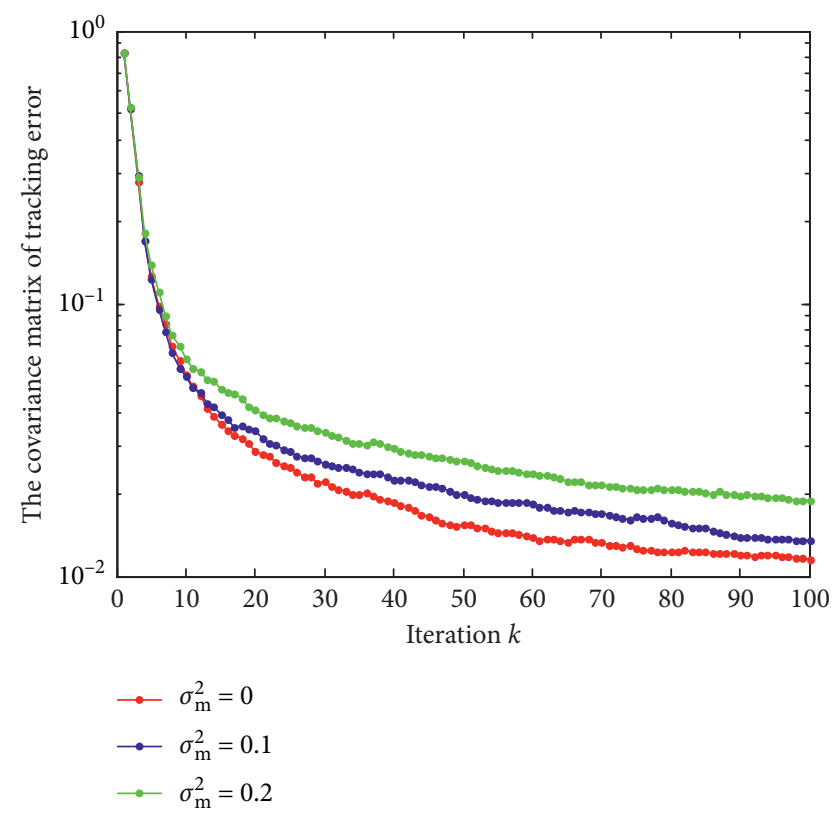

FIGURE 6: Convergence speed of the covariance matrix of tracking error with different variances of channel noise.

tracking error, and the smaller the rates, the faster the convergence speed. Nonetheless, the convergence of tracking error can be guaranteed.

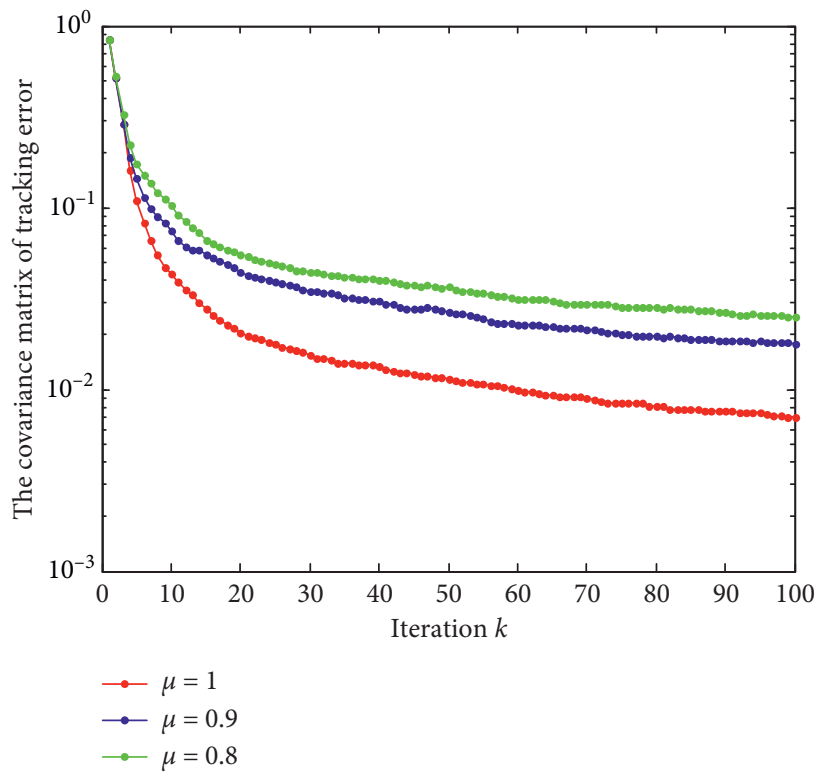

FIGURE 7: Convergence speed of the covariance matrix of tracking error with different successful transfer rates.

\section{Conclusions}

This paper deals with the consensus problem of multiagent systems over wireless networks in the presence of channel noise and data dropout via an average-ILC approach. The gain matrix is designed to satisfy sufficient conditions. Rigorous theories demonstrate that the convergence of the covariance matrix of tracking error can be guaranteed with the help of an average-iterative learning scheme. To highlight the effectiveness of the proposed method, a benchmark method is realized to be a reference in Section 5. As a result, it is proved that convergence performance of the proposed method is much better than the benchmark one, and perfect tracking in the sense of expectation can be realized asymptotically. In the future work, consensus problems for a class of MAS with unknown dynamics will be discussed. Then, channel noise, data dropout, and other disturbances in communication channels are also considered.

\section{Data Availability}

The data used to support the findings of this study are available from the corresponding author upon request.

\section{Conflicts of Interest}

The authors declare that they have no conflicts of interest. 


\section{Acknowledgments}

The work was supported by National Natural Science Foundation of China (NSFC) under Grant nos. 61673253 and 61901254.

\section{References}

[1] H. Zhang, J. Zhang, G.-H. Yang, and Y. Luo, "Leader-based optimal coordination control for the consensus problem of multiagent differential games via fuzzy adaptive dynamic programming," IEEE Transactions on Fuzzy Systems, vol. 23, no. 1, pp. 152-163, 2015.

[2] M. Saim, S. Ghapani, W. Ren, K. Munawar, and U. M. AlSaggaf, "Distributed average tracking in multi-agent coordination: extensions and experiments," IEEE Systems Journal, vol. 12, no. 3, pp. 2428-2436, 2018.

[3] D. Meng and K. L. Moore, "Studies on resilient control through multiagent consensus networks subject to disturbances," IEEE Transactions on Cybernetics, vol. 44, no. 11, pp. 2050-2064, 2014.

[4] D. Meng, M. Du, and Y. Jia, "Interval bipartite consensus of networked agents associated with signed digraphs," IEEE Transactions on Automatic Control, vol. 61, no. 12, pp. 3755-3770, 2016.

[5] S. Sun, T. Endo, and F. Matsuno, "Iterative learning control based robust distributed algorithm for non-holonomic mobile robots formation," IEEE Access, vol. 6, pp. 61904-61917, 2018.

[6] W. He, T. Meng, X. He, and C. Sun, "Iterative learning control for a flapping wing micro aerial vehicle under distributed disturbances," IEEE Transactions on Cybernetics, vol. 49, no. 4, pp. 1524-1535, 2019.

[7] S. Bifaretti, P. Tomei, and C. M. Verrelli, "A global robust iterative learning position control for current-fed permanent magnet step motors," Automatica, vol. 47, no. 1, pp. 227-234, 2011.

[8] D. Li, S. He, Y. Xi et al., "Synthesis of ILC-MPC controller with data-driven approach for constrained batch processes," IEEE Transactions on Industrial Electronics, vol. 67, no. 4, pp. 3116-3125, 2020.

[9] H. S. Ahn and Y. Chen, "Iterative learning control for multiagent formation," in Proceedings of the 2009 ICCAS-SICE, pp. 3111-3116, Fukuoka, Japan, Augest 2009.

[10] Y. Liu and Y. Jia, "Formation control of discrete-time multiagent systems by iterative learning approach," International Journal of Control, Automation and Systems, vol. 10, no. 5, pp. 913-919, 2012.

[11] Y. Liu and Y. Jia, "An iterative learning approach to formation control of multi-agent systems," Systems \& Control Letters, vol. 61, no. 1, pp. 148-154, 2012.

[12] D. Meng and Y. Jia, "On iterative learning algorithms for the formation control of nonlinear multi-agent systems," Automatica, vol. 50, no. 1, pp. 291-295, 2013.

[13] S. Devasia, "Iterative control for networked heterogeneous multi-agent systems with uncertainties," IEEE Transactions on Automatic Control, vol. 62, no. 1, pp. 431-437, 2017.

[14] Xu JIN, "Nonrepetitive leader-follower formation tracking for multiagent systems with LOS range and angle constraints using iterative learning control," IEEE Transactions on Cybernetics, vol. 49, no. 5, pp. 1748-1758, 2018.

[15] D. Meng, W. Du, and Y. Jia, "Data-driven consensus control for networked agents: an iterative learning control-motivated approach," IET Control Theory \& Applications, vol. 9, no. 14, pp. 2084-2096, 2015.
[16] Y. Ren and Z. Hou, "Robust model-free adaptive iterative learning formation for unknown heterogeneous non-linear multi-agent systems," IET Control Theory \& Applications, vol. 14, no. 4, pp. 654-663, 2020.

[17] X. Bu, J. Liang, Z. Hou, and R. Chi, "Data-driven terminal iterative learning consensus for nonlinear multiagent systems with output saturation," IEEE Transactions on Neural Networks and Learning Systems, vol. 32, no. 5, pp. 1963-1973, 2020.

[18] X. Bu, Z. Hou, Q. Yu, and Y. Yang, "Quantized data driven iterative learning control for a class of nonlinear systems with sensor saturation," IEEE Transactions on Systems, Man, and Cybernetics: Systems, vol. 50, no. 12, pp. 5119-5129, 2020.

[19] X. Bu, P. Zhu, Z. Hou, and J. Liang, "Finite-time consensus for linear multi-agent systems using data-driven terminal ILC," IEEE Transactions on Circuits and Systems II: Express Briefs, vol. 67, no. 10, pp. 2029-2033, 2020.

[20] J. Mao, Y. Sun, X. Yi, H. Liu, and D. Ding, "Recursive filtering of networked nonlinear systems: a survey," International Journal of Systems Science, vol. 52, no. 6, pp. 1110-1128, 2021.

[21] X. Ge, Q.-L. Han, L. Ding, Y.-L. Wang, and X.-M. Zhang, "Dynamic event-triggered distributed coordination control and its applications: a survey of trends and techniques," IEEE Transactions on Systems, Man, and Cybernetics: Systems, vol. 50, no. 9, pp. 3112-3125, 2020.

[22] D. Ding, Q.-L. Han, X. Ge, and J. Wang, "Secure state estimation and control of cyber-physical systems: a survey," IEEE Transactions on Systems, Man, and Cybernetics: Systems, vol. 51, no. 1, pp. 176-190, 2021.

[23] L. Li, Y. Liu, Z. Yang, X. Yang, and K. Li, “Mean-square error constrained approach to robust stochastic iterative learning control," IET Control Theory \& Applications, vol. 12, no. 1, pp. 38-44, 2018.

[24] L. Li, Y. Liu, L. Li, and J. Tan, "Kalman-filtering-based iterative feedforward tuning in presence of stochastic noise: with application to a wafer stage," IEEE Transactions on Industrial Informatics, vol. 15, no. 11, pp. 5816-5826, 2019.

[25] L. x. Huang, Y. Fang, and T. Wang, "Method to improve convergence performance of iterative learning control systems over wireless networks in presence of channel noise," IET Control Theory \& Applications, vol. 8, no. 3, pp. 175-182, 2014.

[26] B. Li, H. Lin, and H. Xing, "Adaptive adjustment of iterative learning control gain matrix in Harsh noise environment," Journal of Systems Engineering and Electronics, vol. 24, no. 1, pp. 128-134, 2013.

[27] Y. Geng, X. Ruan, and J. Xu, "Adaptive iterative learning control of switched nonlinear discrete-time systems with unmodeled dynamics," IEEE Access, vol. 7, pp. 118370118380, 2019.

[28] D. Shen and G. Qu, "Performance enhancement of learning tracking systems over fading channels with multiplicative and additive randomness," IEEE Transactions on Neural Networks and Learning Systems, vol. 31, no. 4, pp. 1196-1210, 2020.

[29] L. Huang, Q. Zhang, L. Sun, and Z. Sheng, "Robustness analysis of iterative learning control for a class of mobile robot systems with channel noise," IEEE Access, vol. 7, pp. 3471134718, 2019.

[30] Y. Wu, B. Hu, and Z.-H. Guan, "Consensus problems over cooperation-competition random switching networks with noisy channels," IEEE Transactions on Neural Networks and Learning Systems, vol. 30, no. 1, pp. 35-43, 2019.

[31] L. Huang, Q. Zhang, W. Liu, J. Li, L. Sun, and T. Wang, "Convergence analysis of iterative learning control systems 
over networks with successive input data compensation in iteration domain," IEEE Access, vol. 7, pp. 160217-160226, 2019.

[32] D. Shen and J.-X. Xu, "A novel Markov chain based ILC analysis for linear stochastic systems under general data dropouts environments," IEEE Transactions on Automatic Control, vol. 62, no. 11, pp. 5850-5857, 2017.

[33] D. Shen, "Data-driven learning control for stochastic nonlinear systems: multiple communication constraints and limited storage," IEEE Transactions on Neural Networks and Learning Systems, vol. 29, no. 6, pp. 2429-2440, 2018.

[34] T. Zhang and J. Li, "Iterative learning control for multi-agent systems with finite-leveled sigma-delta quantization and random packet losses," IEEE Transactions on Circuits and Systems I: Regular Papers, vol. 64, no. 8, pp. 2171-2181, 2017.

[35] M. Sun, T. Wu, L. Chen, and G. Zhang, "Neural AILC for error tracking against arbitrary initial shifts," IEEE Transactions on Neural Networks and Learning Systems, vol. 29, no. 7, pp. 2705-2716, 2018. 